\title{
Refractory Shock from Amlodipine Overdose Overcomed with Hyperinsulinemia
}

\author{
L. Koliastasis $^{1}$ D $\cdot$ I. Lampadakis ${ }^{1} \cdot$ A. Milkas ${ }^{1,2} \cdot$ P. Strempelas ${ }^{1} \cdot$ V. Sourides ${ }^{1} \cdot$ K. Kakava ${ }^{1} \cdot$ P. Tsioufis ${ }^{1}$. \\ S. Papaioannou ${ }^{1}$
}

Received: 2 June 2021 / Accepted: 27 September 2021 / Published online: 13 October 2021

(c) The Author(s), under exclusive licence to Springer Science+Business Media, LLC, part of Springer Nature 2021

\begin{abstract}
Intoxication from calcium channel blockers exhibits almost $50 \%$ mortality rates. Amlodipine is a long-acting dihydropyridine and inappropriate dosage poses a great threat for profound vasodilation, hypotension, and refractory vasopressor-resistant shock. A 72-year-old woman with unremarkable medical history presented to the emergency department due to amlodipine overdose after a suicide attempt attributed to COVID-19 pandemic severe anxiety disorder. Vital signs at presentation: heart rate 82 beats/ min, arterial pressure $72 / 55 \mathrm{mmHg}$, and oxygen saturation $98 \%$. Resuscitation was initiated with intravenous infusion of normal saline $0,9 \%$, noradrenaline, and calcium chloride, while activated charcoal was orally administrated; however, blood pressure remained at $70 / 45 \mathrm{mmHg}$. Abruptly, she experienced acute pulmonary edema and was finally intubated. We commenced high-dose insulin infusion with Dextrose $10 \%$ infusion to maintain euglycemic hyperinsulinemia. Hemodynamic improvement occurred after $30 \mathrm{~min}$, systolic blood pressure raised to $95 \mathrm{mmHg}$, and decongestion was achieved with intravenous furosemide. Insulin effect was dose-dependent and patient's hemodynamic status improved after insulin uptitration. Eight days later, the patient was weaned from the mechanical ventilation and she was successfully discharged after 14 days. High-dose intravenous infusion of insulin up to 10 units/kg per hour appears as an inotropic agent possibly through alterations in myocardial metabolism of fatty acids and augmentation of insulin secretion and uptake. This regimen possibly exhibits additional vasotropic properties. We conclude that euglycemic hyperinsulinemia is a potentially advantageous treatment in CCB toxicity.
\end{abstract}

Keywords Amlodipine $\cdot$ Overdose $\cdot$ Hyperinsulinemia $\cdot$ Insulin $\cdot$ Calcium channel blocker

$\begin{array}{ll}\text { Abbreviations } \\ \text { ACCU } & \text { Acute Cardiac Care Unit } \\ \text { CCB } & \text { Calcium Channel Blockers } \\ \text { COVID-19 } & \text { COronaVIrus Disease 2019 } \\ \text { ECMO } & \text { Extracorporeal Membrane Oxygenation } \\ \text { ED } & \text { Emergency Department } \\ \text { NS } & \text { Normal Saline } 0,9\end{array}$

Handling Editor: Rajiv Janardhanan.

L. Koliastasis and I. Lampadakis have contributed equally to this work

L. Koliastasis

lkoliastasis@gmail.com

1 Cardiology Department, Athens Naval and Veterans Hospital, Mikras Asias 48, 11257 Athens, Greece

2 Acute Cardiac Care Unit, Athens Naval and Veterans Hospital, Athens, Greece

\section{Introduction}

Calcium channel blockers (CCB) intoxication is a leading cause of cardiovascular medication overdose exhibiting high-almost 50\%—-mortality rates [1]. Amlodipine is a long-acting dihydropyridine derivative with a half-life of 30-50 h [2]. Inappropriate dosage poses a great threat due to profound vasodilation, hypotension, and refractory vasopressor-resistant shock. High-dose insulin infusion has been described as a potential shock reversal agent induced by CCB poisoning [3].

We describe a clinical case of a 72-year-old woman effectively treated with euglycemic hyperinsulinemia after developing refractory shock due to amlodipine overdose. 


\section{Case Presentation}

A 72-year-old woman with unremarkable medical history presented to the emergency department (ED) due to amlodipine overdose ( $200 \mathrm{mg}$ of amlodipine by oral route) after a suicide attempt three hours ago. The incident was attributed to severe anxiety disorder arising from the pandemic of coronavirus disease 2019 (COVID-19). Vital signs at presentation: heart rate 82 beats/ min, arterial pressure $72 / 55 \mathrm{mmHg}$, oxygen saturation $98 \%$, and temperature at $36.4{ }^{\circ} \mathrm{C}$. Physical findings were normal and she was oriented and fluent. Electrocardiogram demonstrated that sinus rhythm and cardiac ultrasound was within normal limits.

Resuscitation was initiated with intravenous Normal Saline $0.9 \%$ (NS) and $60 \mathrm{gr}(1 \mathrm{gr} / \mathrm{kg})$ of activated charcoal was orally administrated to inhibit the absorption of amlodipine. She was transferred to the Acute Cardiac Care Unit (ACCU) due to shortage of beds in the Intensive Care Unit during the first wave of COVID-19 outbreak in Europe. Within the first hours of ACCU monitoring blood pressure decreased at $70 / 50 \mathrm{mmHg}$. Intravenous noradrenaline infusion was initiated with gradual uptitration to $1 \mathrm{mcg} / \mathrm{kg} / \mathrm{min}$. We introduced simultaneously calcium chloride $10 \%$ infusion at $15 \mathrm{mg} / \mathrm{hr}$ in an attempt to overcome the competitive blockage of calcium channels. After administrating $2 \mathrm{~L}$ of NS $(11 \mathrm{ml} / \mathrm{kg} / \mathrm{hr})$ and increasing noradrenaline dose at $2.5 \mathrm{mcg} / \mathrm{kg} / \mathrm{min}$, blood pressure decreased further at $65 / 45 \mathrm{mmHg}$. Abruptly, she experienced dyspnea with mental status deterioration and signs of acute pulmonary edema provoked by a combination of iatrogenic congestion with amlodipine vasoplegia and led to intubation and mechanical ventilation.
We commenced high-dose insulin infusion (45 IU/ hr) alongside with intravenous $10 \%$ dextrose solution to maintain euglycemia. After $30 \mathrm{~min}$, systolic blood pressure elevated to $95 \mathrm{mmHg}$ and the patient was treated with intravenous furosemide to gradually regularize volemia and oppose organ congestion. Further insulin uptitration to $60 \mathrm{IU} / \mathrm{hr}(1 \mathrm{IU} / \mathrm{kg} / \mathrm{hr})$ was followed by systolic blood pressure augmentation at $105 \mathrm{mmHg}$.

Calcium chloride infusion was terminated 3 days later, insulin infusion after 5 days, as well as noradrenaline. Gradual downtitration of insulin and noradrenaline was driven by the patient's hemodynamic status. It is remarkable that attempts to reduce the dose of insulin during the first 3 days were followed by immediate decrease of blood pressure; insulin effect was dose-dependent (Fig. 1). Eight days later, the patient was weaned from mechanical ventilation. She was discharged after 14 days. Cardiac function tests were unremarkable and she received antipsychotic treatment. One-month clinical and laboratory follow-up was performed and the patient exhibited improved mental status with no cardiovascular comorbidity.

\section{Discussion}

CCB act at the L-type calcium channels in heart and vessels resulting in vasodilation and reduction of both inotropism and chronotropism. Effects of CCB in pancreas include inhibition of insulin secretion and favored insulin resistance with consequent reduced glucose uptake by cardiac and vascular myocytes (the leading factor of CCB cardiovascular toxicity) [4]. Regarding the gastrointestinal tract, motility alterations and possible increase of bleeding have been described
Fig. 1 Systolic blood pressure (mmHg) fluctuation during insulin administration. $0 \mathrm{~h}$ (hrs) represents the initiation of insulin infusion

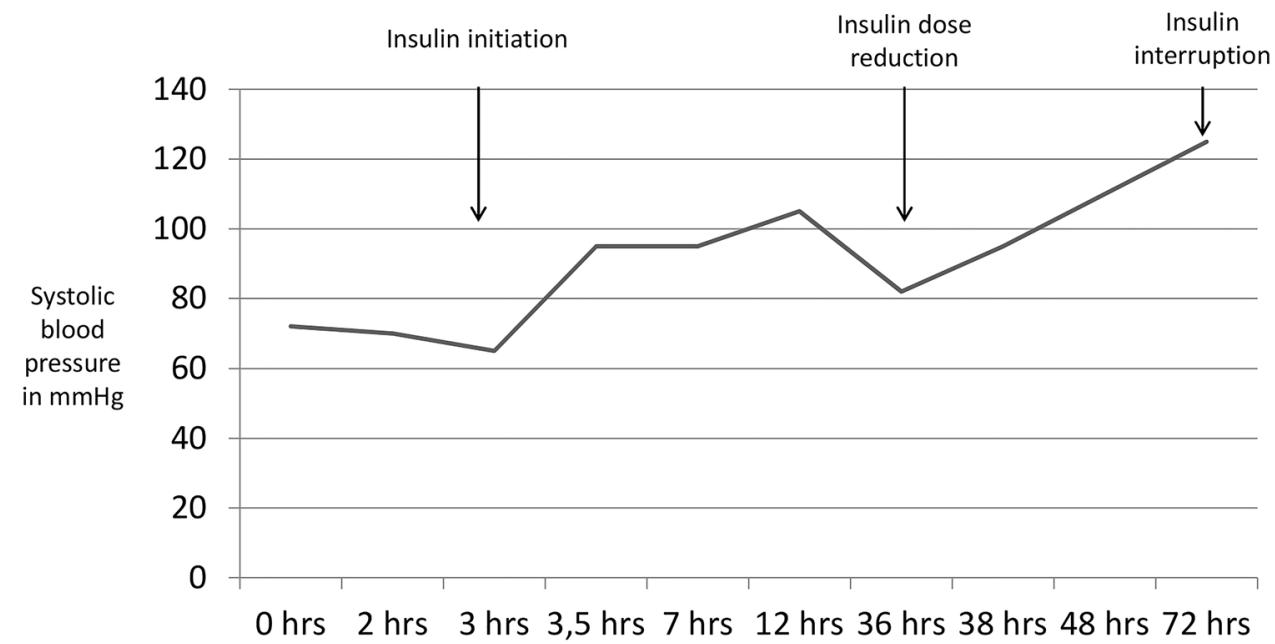

Hours after insulin infusion initiation 
during CCB administration [5]. CCB exhibit metabolic effects as well by modifying metabolism and kinetics of beta-adrenergic receptors and cardiac glycosides [6]. Dihydropyridines have a predominant effect on vascular motility and do not interfere with cardiac conduction properties, pacemaker cells, and contractility. This selectivity, however, fades in significant overdose resulting in bradycardia, conduction abnormalities, and reduced inotropism (Fig. 2) [1].

The patient presented in the ED as hypotensive and asymptomatic, nevertheless, the situation rapidly evolved in refractory shock. Noradrenaline, fluids, and calcium exerted no clinical effect and hypotension was followed by respiratory insufficiency and mental status alteration in the context of acute pulmonary edema. The latter is a frequent complication of CCB overdose and the most dominant pathophysiological pathway is massive pulmonary capillary dilatation and increased permeability, possibly sharing a similar mechanism as peripheral edema caused by pre-capillary vasodilatation [7].

The CCB-induced shock is vasogenic and first-line management includes fluid resuscitation and vasopressors. High intravenous calcium doses as calcium chloride or calcium gluconate may be thought to antagonize the CCB toxicity by increasing serum ionic calcium level enough to overcome calcium channels blockade and drive calcium into the cells. Atropine and cardiac pacing are required in the cases of bradycardia and conduction abnormalities. Lipid emulsion, methylene blue, and glucagon are included in our quiver as additional alternative therapeutic approaches, and ECMO (Extracorporeal membrane oxygenation) has been described as a potential bridging therapy until the involved drug has been metabolized [8]. CCB are strongly bounded to plasma proteins so that conventional hemodialysis is not effective. Gastrointestinal decontamination with gastric lavage, whole-bowel irrigation, and activated charcoal remains the only option for preventing the drugs' absorption, but should be ideally administered shortly after ingestion [9].

CCB plasma levels correlate directly with increased serum glucose concentrations primarily because they block pancreatic L-type calcium channels leading to inhibition of insulin release (10). This "diabetogenic" effect triggered investigation on $\mathrm{CCB}$ toxicity monitoring through measuring serum glucose levels [11]. Case reports and animal studies suggest that hyperinsulinemia improves hemodynamics in CCB overdose. The dose varies among 1-10 units $/ \mathrm{kg} / \mathrm{hour}$, while glucose and potassium levels should be meticulously monitored and corrected [12]. Some reports support a loading bolus dose of insulin followed by maintenance infusion and other infusion drip only for adequate control of blood pressure and end organs perfusion [13]. Hyperinsulinemia acts through improving cardiac contractility by overcoming carbohydrates' starvation and therefore providing heart's metabolic demands [14]. Experimental models demonstrated that insulin accelerates myocardial lactates oxidation and facilitates calcium handling from myocardial cells. The
Fig. 2 Calcium Channel Blockers toxicity effects

\section{Calcium channel blocker toxicity}


Vessels

- Vasodilation (decreased vascular tone)

- Insulin resistance

- Decreased afterload

\section{Gastrointestinal tract}

- Motility alterations

- Increased risk of bleeding

\section{Pancreas}

- Insulin secretion inhibition

\author{
Heart \\ - Insulin resistance \\ - Reduced \\ inotropism/chronotropism \\ (non-dihydropyridines)
}


aforementioned effects render insulin as an effectual inotrope, even better than epinephrine which increases oxygen consumption [15].

In our case report, we exhibited that euglycemic hyperinsulinemia was the key to stabilize the patient in accordance to existing literature. As the dose regimen has not been well established, we commenced the infusion at less than $1 \mathrm{unit} / \mathrm{kg} / \mathrm{hour}$ with gradual uptitration and observed that blood pressure elevation was proportional to the insulin dose. Interestingly, any dose reduction led to blood pressure drop. Even after intubation and concomitant propofol administration, the patient remained stable receiving insulin. Interestingly, our patient's systolic function was normal and remained unaffected during the hospitalization, therefore, there was no need for inotropic support. Based on the hemodynamic stabilization after insulin initiation, we could assume that the insulin exerted pleiotropic vasotropic actions. The effectiveness of the euglycemic hyperinsulinemia possibly renders this regimen as a first-line treatment for CCB toxicity-induced shock.

\section{Conclusion}

We conclude that hyperinsulinemia could be an advantageous treatment for CCB intoxication and possibly included in the first-line regimens. Hemodynamic response is in proportion with insulin administered dose, and for maximal beneficiality, careful uptitration is suggested in conjunction with the conventional treatment.

Funding None.

Data Availability No additional data are available. This case report is supported by the facts and data included in the manuscript.

\section{Declarations}

\section{Conflict of interest None.}

Ethical Approval No ethics approval is required.

Informed Consent Signed consent has been obtained.

Consent for Publication The patient and the authors consent for publication.

\section{References}

1. Ghosh, S., \& Sircar, M. (2008). Calcium channel blocker overdose: Experience with amlodipine. Indian J Crit Care Med., 12(4), 190-193.

2. Bulsara, K. G., \& Cassagnol, M. (2021). Amlodipine. StatPearls.

3. Levine, M. D., \& Boyer, E. (2006). Hyperinsulinemia-euglycemia therapy: A useful tool in treating calcium channel blocker poisoning. Critical Care, 10(4), 149.

4. Tuch, B. E., Osgerby, K. J., \& Turtle, J. R. (1990). The role of calcium in insulin release from the human fetal pancreas. Cell Calcium, 11(1), 1-9.

5. He, Y., Chan, E. W., Leung, W. K., Anand, S., \& Wong, I. C. (2015). Systematic review with meta-analysis: The association between the use of calcium channel blockers and gastrointestinal bleeding. Alimentary Pharmacology \& Therapeutics, 41(12), 1246-1255.

6. Russell, R. P. (1988). Side effects of calcium channel blockers. Hypertension., 11(3 Pt 2), II42-4.

7. Humbert, V. H., Jr., Munn, N. J., \& Hawkins, R. F. (1991). Noncardiogenic pulmonary edema complicating massive diltiazem overdose. Chest, 99(1), 258-259.

8. Graudins, A., Lee, H. M., \& Druda, D. (2016). Calcium channel antagonist and beta-blocker overdose: Antidotes and adjunct therapies. British Journal of Clinical Pharmacology, 81(3), 453-461.

9. Albertson, T. E., Owen, K. P., Sutter, M. E., \& Chan, A. L. (2011). Gastrointestinal decontamination in the acutely poisoned patient. International Journal of Emergency Medicine, 4, 65.

10. Levine, M., Boyer, E. W., Pozner, C. N., Geib, A. J., Thomsen, T., Mick, N., et al. (2007). Assessment of hyperglycemia after calcium channel blocker overdoses involving diltiazem or verapamil. Critical Care Medicine, 35(9), 2071-2075.

11. Mycyk, M. B., \& Bryant, S. M. (2007). Is simple bedside glucose assessment prognostic in calcium channel blocker overdose? Critical Care Medicine, 35(9), 2216-2217.

12. Engebretsen, K. M., Kaczmarek, K. M., Morgan, J., \& Holger, J. S. (2011). High-dose insulin therapy in beta-blocker and calcium channel-blocker poisoning. Clinical Toxicology (Philadelphia, PA), 49(4), 277-283.

13. Levine, M., Curry, S. C., Padilla-Jones, A., \& Ruha, A. M. (2013). Critical care management of verapamil and diltiazem overdose with a focus on vasopressors: A 25-year experience at a single center. Annals of Emergency Medicine, 62(3), 252-258.

14. Kline, J. A., Leonova, E., \& Raymond, R. M. (1995). Beneficial myocardial metabolic effects of insulin during verapamil toxicity in the anesthetized canine. Critical Care Medicine, 23(7), 1251-1263.

15. Megarbane, B., Karyo, S., \& Baud, F. J. (2004). The role of insulin and glucose (hyperinsulinaemia/euglycaemia) therapy in acute calcium channel antagonist and beta-blocker poisoning. Toxicological Reviews, 23(4), 215-222.

Publisher's Note Springer Nature remains neutral with regard to jurisdictional claims in published maps and institutional affiliations. 